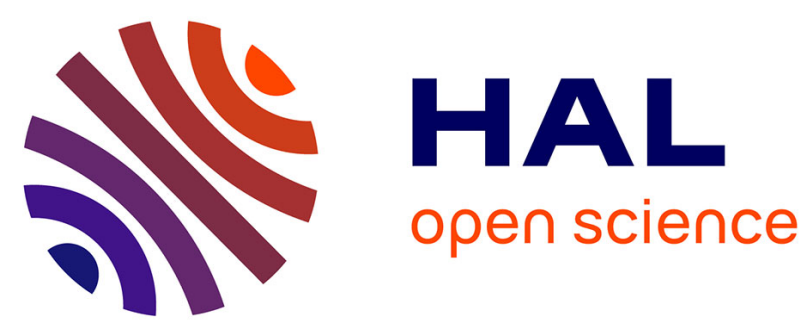

\title{
Modelling and Analysis of Multi-Stage Systems of Mistuned Bladed Disks
}

Denis Laxalde, Christophe Pierre

\section{To cite this version:}

Denis Laxalde, Christophe Pierre. Modelling and Analysis of Multi-Stage Systems of Mistuned Bladed Disks. Computers \& Structures, 2011, 89 (3-4), pp.316-324. 10.1016/j.compstruc.2010.10.020 . hal$00468477 \mathrm{v} 2$

\section{HAL Id: hal-00468477 \\ https://hal.science/hal-00468477v2}

Submitted on 7 Jan 2011

HAL is a multi-disciplinary open access archive for the deposit and dissemination of scientific research documents, whether they are published or not. The documents may come from teaching and research institutions in France or abroad, or from public or private research centers.
L'archive ouverte pluridisciplinaire HAL, est destinée au dépôt et à la diffusion de documents scientifiques de niveau recherche, publiés ou non, émanant des établissements d'enseignement et de recherche français ou étrangers, des laboratoires publics ou privés. 


\title{
Modelling and Analysis of Multi-Stage Systems of Mistuned Bladed Disks
}

\author{
Denis LAXALDE and Christophe PIERRE \\ Structural Dynamics and Vibration Laboratory \\ Department of Mechanical Engineering \\ McGill University, Montreal, QC, Canada
}

\begin{abstract}
A stochastic reduced-order modelling technique for multi-stage mistuned bladed disks assemblies is proposed. In a perturbation framework, the base-line reduced-order model is built using multi-stage cyclic symmetry modelling approach. Uncertainties are introduced in the modal space at the component level and further propagated in the global modal space. This results in a compact model suitable for statistical analyses based on Monte-Carlo simulations.

Applications highlight the numerically efficiency of the proposed approach which features good correlation and convergence both from deterministic and probabilistic points of view. Statistical investigations on the forced response amplification show effects of multi-stage coupling on mistuning sensitivity.

Keywords: cyclic symmetry; mistuning; multi-stage; uncertainties; reduce-order models
\end{abstract}

\section{Introduction}

Accounting for uncertainties in mechanical systems is now commonly recognized as an important part of a successful design process. Structural uncertainty may be due to numerous factors such as material inhomogeneity, manufacturing processes, environment conditions, variability in configurations, etc. Uncertainty analyses are usually based on probabilistic methods and involve quantification of uncertainties followed by a propagation to the system response via the system model. When it comes to complex dynamical systems, which require high fidelity and large-scale models and can feature complicated and rich dynamics, several difficulties may appear, among which: (a) computational efforts required when using stochastic solvers and (b) quantification of uncertainties in complex computational models.

In turbine engines rotating components (bladed disks), one of the main sources of structural uncertainty is mistuning. Mistuning refers to variations in the characteristics of each sector of a system ideally expected to be cyclically symmetric [17]. It generally results in some variability of dynamical responses and induces localization phenomena and stress magnification $[18,19]$. Over the past decades, this issue has motivated numerous research works which have focused on several aspects such as modelling methodologies, statistical analysis, experimental identification or inclusion of other phenomenological issues (such as friction dissipation or aerodynamic coupling). Recent surveys [6, 15] provide extensive reviews of the question and discuss research advances and trends. Concerning methodological aspects, it is noticeable that the use of reduced-order modelling techniques based on modal synthesis has been made quite systematic. Among other advantages [8], this framework offers a common answer to the two previously mentioned issues concerning complex systems as (a) computational costs are drastically reduced and (b) uncertainties can be quantified directly in the reduced space. While early works involved component mode synthesis approaches (see [1] for example), more recent techniques [13, 20] no longer require sub-structuring and are only based on perturbational modal synthesis leading to even more compact models.

One of the remaining issues [6] concerns the modelling and analysis of mistuning phenomena in multistage systems of bladed disks. Indeed, in modern turbine engines, which feature improved design and 
optimisation, lightweight material and increased aerodynamic load, occurrences of multi-stage dynamical phenomena tend to become rather ordinary so that modelling of multi-stage can be regarded as a challenge in itself. Recently [10, 11], an extension of the cyclic symmetry [17] approach to multi-stage systems was proposed, thus overcoming one the fundamental obstacle related to this subject. The latter involves the modelling of a single sector per stage, making direct finite-element analyses affordable and features a high level of accuracy. As multi-stage analyses can now be conducted within traditional design processes, a natural extension would be to consider mistuning in this framework.

This paper proposes a reduced-order modelling strategy for multi-stage system of mistuned bladed disks that is suitable for the propagation of uncertainties defined in the modal space of local components to the global system dynamic response. The first three sections are devoted to (1) the derivation of the system's equations of the motion and assembly, (2) cyclic symmetry and multi-stage cyclic symmetry formulations and (3) description of the stochastic reduced-order model and discussions about quantification of uncertainties. Besides, a two-stage HP compressor model is considered as an application. The methodology is validated both from deterministic and statistic points of view. Then, mistuning sensitivity analyses are performed to illustrate the importance of proper assessment multi-stage coupling in mistuning analyses.

\section{Multi-stage systems: equations of motion and coupling}

Consider a multi-stage system of $S$ cyclic structures sharing the same axis of rotational symmetry. In absence of mistuning, each sub-structure $s$ is rotationally periodic, that is, composed of $N_{s}$ identical sectors (Fig. 1a). Each sector $\Omega_{n}$ is obtained by $n$ rotations of the fundamental (or reference) sector $\Omega_{0}$ by the angle $2 \pi / N_{s}$

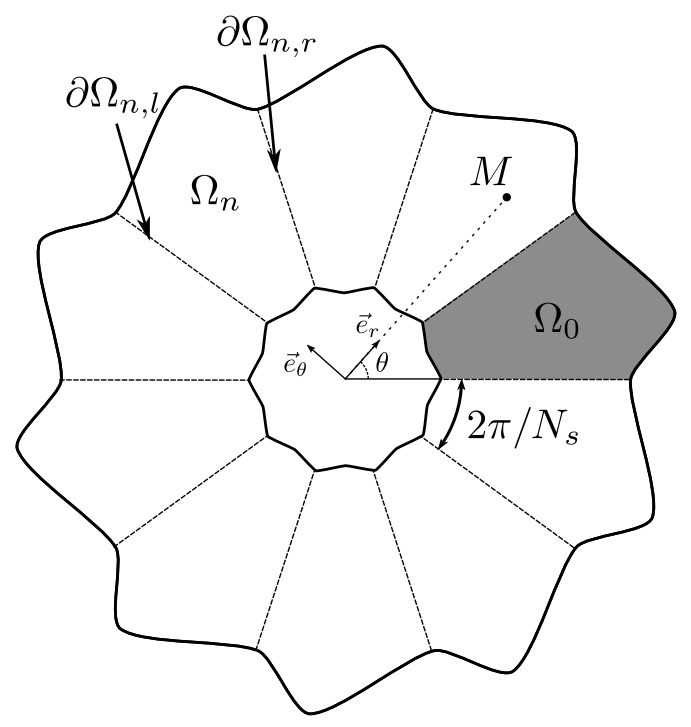

(a) Cyclic structure

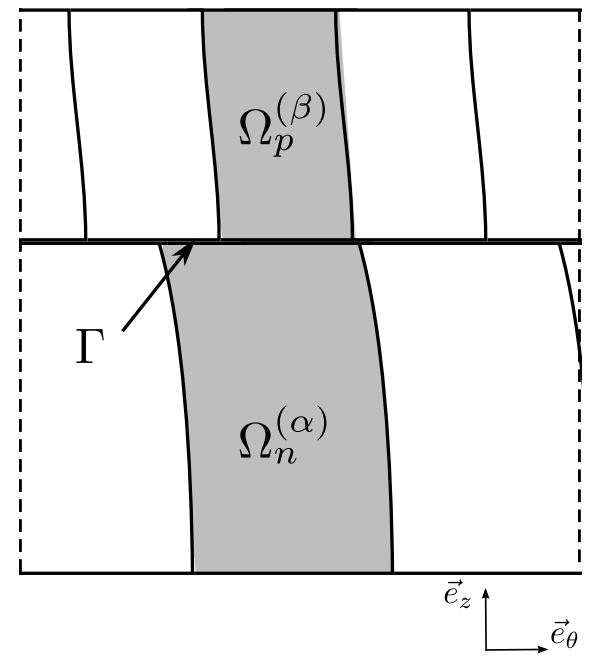

(b) Multi-stage interface

Figure 1: Description of inter-sector and inter-stage interfaces for cyclic structures

about the axis of rotational symmetry $(O, \boldsymbol{z}): \theta_{n}=\theta_{0}+2 \pi n / N_{s}$.

The equations of motion of each $s$ sub-structure (i.e. stage) may be written as

$$
\boldsymbol{M}^{(s)} \ddot{\boldsymbol{u}}^{(s)}+\boldsymbol{C}^{(s)} \dot{\boldsymbol{u}}^{(s)}+\boldsymbol{K}^{(s)} \boldsymbol{u}^{(s)}=\boldsymbol{f}^{(s)}
$$

where $\boldsymbol{M}^{(s)}, \boldsymbol{C}^{(s)}$ and $\boldsymbol{K}^{(s)}$ are the structural mass, damping and stiffness matrices of sub-structure $s, \boldsymbol{u}^{(s)}$ is its vector of degrees-of-freedom and $\boldsymbol{f}^{(s)}$ is an external forcing vector. Vectors $\boldsymbol{u}^{(s)}$ and $\boldsymbol{f}^{(s)}$ consist of the concatenation of each sector's characteristic vectors $\boldsymbol{u}^{(s, n)}$ and $\boldsymbol{f}^{(s, n)}$ for $n \in \llbracket 0, N_{s}-1 \rrbracket$. 
The multi-stage assembly is defined by a set of inter-stage compatibility conditions on each interface $\Gamma$ between stages $\alpha$ and $\beta$

$$
\left(\mathcal{I}_{\alpha} \otimes \boldsymbol{B}_{\Gamma, \alpha}\right) \boldsymbol{u}^{(\alpha)}-\left(\mathcal{I}_{\beta} \otimes \boldsymbol{B}_{\Gamma, \beta}\right) \boldsymbol{u}^{(\beta)}=0
$$

in which $\otimes$ denotes the Kronecker product and matrices $\boldsymbol{B}_{\Gamma, \alpha}$ and $\boldsymbol{B}_{\Gamma, \beta}$ operate on boundary degrees-offreedom of an elementary sector of stages $\alpha$ and $\beta$ respectively along their common interface $\Gamma$ (see Fig. 1b). These matrices have zero entries corresponding to internal degrees-of-freedom and we denote $\overline{\boldsymbol{B}}_{\Gamma, \alpha}$ and $\overline{\boldsymbol{B}}_{\Gamma, \beta}$ the sub-matrices of the latter restricted to interfaces degrees-of-freedom along boundary $\Gamma$. In case of compatible interfaces (conforming meshes), these matrices are Boolean, otherwise they can be derived from application of multipoint constraints. Finally, note that a primal assembly of sub-structures is here considered so that connecting forces have been omitted from the equations of motion (1) as they would eventually have been eliminated during the assembly.

Multi-stage cyclic symmetry $[10,11]$ is based on the modelling of individual stages in (traditional) cyclic symmetry and features a specific interstage coupling defined in a common physical base assuming a congruence of wave numbers. Despite its approximate nature, this method has demonstrated highly satisfying results as compared to full $\left(360^{\circ}\right)$ reference calculations. The following section concerns a review of this method: notations referring to cyclic symmetry modelling are first recalled, then the definition of inter-stage coupling will be detailed. This definition differs from the one initially proposed since it overcomes the issue of aliasing in "higher order" harmonics.

\section{Cyclic symmetry and multi-stage cyclic symmetry}

\subsection{Cyclic symmetry}

Taking advantage of the periodicity of cyclic structures, one may represent any characteristic quantity (displacement, load, etc.) in a so-called cyclic basis using an appropriate discrete Fourier transform. Considering for example the vector of degrees-of-freedom, the so-called cyclic components $\hat{\boldsymbol{u}}_{j}^{(s)}$ (associated with harmonic $\left.j \in \llbracket 0, N_{s}-1 \rrbracket\right)$ are related to physical components by

$$
\hat{\boldsymbol{u}}_{j}^{(s)}=\sum_{n=0}^{N_{s}-1} \mathrm{e}^{-\mathrm{i} \frac{2 \pi j n}{N_{s}}} \boldsymbol{u}^{(s, n)} \quad \text { and } \quad \boldsymbol{u}^{(s, n)}=\frac{1}{N_{s}} \sum_{j=0}^{N_{s}-1} \mathrm{e}^{\mathrm{i} \frac{2 \pi j n}{N_{s}}} \hat{\boldsymbol{u}}_{j}^{(s)}
$$

or, introducing Fourier vectors $\boldsymbol{w}_{s}^{j}=\left(1, \mathrm{e}^{-\mathrm{i} 2 \pi j / N_{s}}, \ldots, \mathrm{e}^{-\mathrm{i} 2 \pi j\left(N_{s}-1\right) / N_{s}}\right)$, in a more compact form:

$$
\hat{\boldsymbol{u}}^{(s)}=\sum_{n=0}^{N_{s}-1} \boldsymbol{w}_{s}^{j} \otimes \boldsymbol{u}^{(s, n)} \quad \text { and } \quad \boldsymbol{u}^{(s)}=\frac{1}{N_{s}} \sum_{j=0}^{N_{s}-1} \boldsymbol{w}_{s}^{j^{*}} \otimes \hat{\boldsymbol{u}}_{j}^{(s)}
$$

where $\hat{\boldsymbol{u}}^{(s)}$ is the vector which concatenates cyclic components vectors $\hat{\boldsymbol{u}}_{j}^{(s)}$ and the superscript $\cdot^{*}$ refers to conjugate-transpose.

In this cyclic basis, Eq. (1) can be re-written as $N_{s}$ sub-problems

$$
\boldsymbol{M}_{j}^{(s)} \ddot{\hat{\boldsymbol{u}}}_{j}^{(s)}+\boldsymbol{C}_{j}^{(s)} \dot{\hat{\boldsymbol{u}}}_{j}^{(s)}+\boldsymbol{K}_{j}^{(s)} \hat{\boldsymbol{u}}_{j}^{(s)}=\hat{\boldsymbol{f}}_{j}^{(s)}
$$

in which cyclic matrices $\boldsymbol{M}_{j}^{(s)}, \boldsymbol{C}_{j}^{(s)}$ and $\boldsymbol{K}_{j}^{(s)}$ are obtained from the matrices of the reference sector to which cyclic boundary conditions have been applied. The latter consists, for harmonic $j$, of a constraint equation between $\boldsymbol{u}_{l}^{(s, 0)}$ and $\boldsymbol{u}_{r}^{(s, 0)}$, the restrictions of the reference sector degrees-of-freedom vector to left and right boundaries with adjacent sectors (see Fig. 1a): $\boldsymbol{u}_{l}^{(s, 0)}=\boldsymbol{u}_{r}^{(s, 0)} \mathrm{e}^{\mathrm{i} 2 \pi j / N_{s}}$. 


\subsection{Multi-stage coupling in cyclic domain}

The definition of the inter-stage coupling is the crucial point of the multi-stage cyclic symmetry method. Using Eqs. (3), the inter-stage displacements compatibility equation (2) can be re-written as

$$
\frac{1}{N_{\alpha}} \sum_{j_{\alpha}=0}^{N_{\alpha}-1} \boldsymbol{w}_{\alpha}^{j_{\alpha}}{ }^{*} \otimes\left(\overline{\boldsymbol{B}}_{\Gamma, \alpha} \hat{\boldsymbol{u}}_{j_{\alpha}}^{(\alpha)}\right)-\frac{1}{N_{\beta}} \sum_{j_{\beta}=0}^{N_{\beta}-1} \boldsymbol{w}_{\beta}^{j_{\beta} *} \otimes\left(\overline{\boldsymbol{B}}_{\Gamma, \beta} \hat{\boldsymbol{u}}_{j_{\beta}}^{(\beta)}\right)=0
$$

in which cyclic harmonics of the two adjacent stages $\hat{\boldsymbol{u}}_{j_{\alpha}}^{(\alpha)}$ and $\hat{\boldsymbol{u}}_{j_{\beta}}^{(\beta)}$ are coupled. For the multi-stage assembly, we define the set of fundamental harmonics with respect to the stage with the smallest number of sectors (stage 1 in our application): $k \in \llbracket 0, N_{1}-1 \rrbracket$. Then, the coupling of Eq. (5) is reduced according to

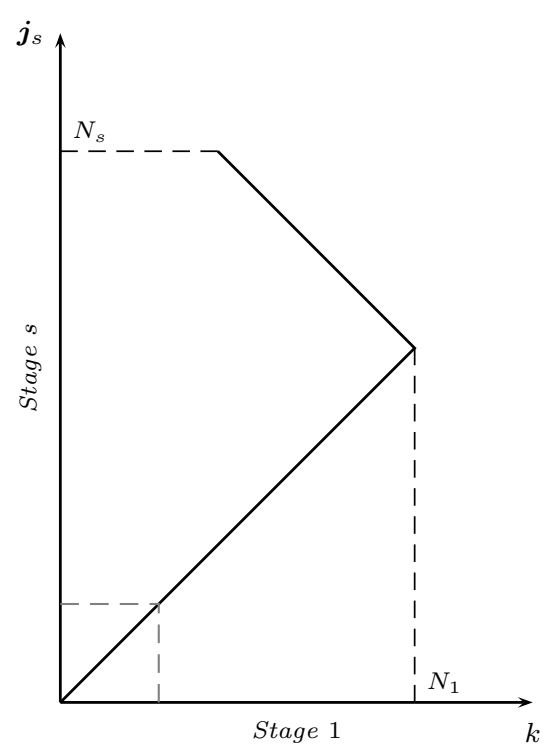

(a)

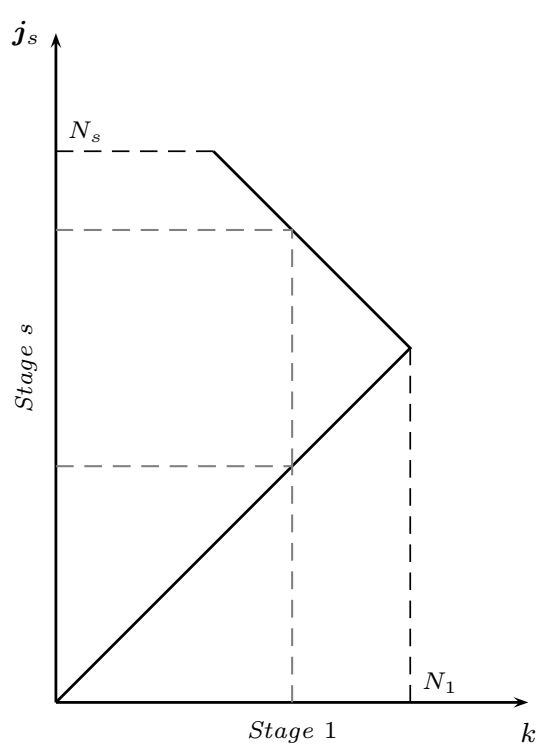

(b)

Figure 2: Cyclic harmonics equivalence rule for multi-stage cyclic assembly; (a) case of small harmonic index $\left(j_{s}=k\right)$ and (b) aliasing case $\left(\boldsymbol{j}_{s}=\left[k, N_{s}-k\right]\right)$.

a congruence rule (illustrated in Figs. 2) which defines a set of cyclic harmonics on stage $s$, denoted $\boldsymbol{j}_{s}$, conformable to the fundamental cyclic harmonic $k$ :

$$
\boldsymbol{j}_{s}=\left\{j_{s, p}=(-1)^{p} k+p N_{s}, p \in \mathbb{N} \mid 0 \leq j_{s, p} \leq N_{s}-1\right\}
$$

This restriction of coupling between equivalent harmonics $k$ and $\boldsymbol{j}_{s}(k)$ means that only compatible harmonic motions are considered. Hence, Eq. (5) is restricted to these compatible harmonics $\boldsymbol{j}_{\alpha}$ and $\boldsymbol{j}_{\beta}$ :

$$
\frac{1}{N_{\alpha}} \sum_{j_{\alpha} \in \boldsymbol{j}_{\alpha}(k)} \boldsymbol{w}_{\alpha}^{j_{\alpha}{ }^{*}} \otimes\left(\overline{\boldsymbol{B}}_{\Gamma, \alpha} \hat{\boldsymbol{u}}_{j_{\alpha}}^{(\alpha)}\right)-\frac{1}{N_{\beta}} \sum_{j_{\beta} \in \boldsymbol{j}_{\beta}(k)} \boldsymbol{w}_{\beta}^{j_{\beta}{ }^{*}} \otimes\left(\overline{\boldsymbol{B}}_{\Gamma, \beta} \hat{\boldsymbol{u}}_{j_{\beta}}^{(\beta)}\right)=0
$$

Finally, if we define Fourier matrices associated with a given set of harmonics $\boldsymbol{h}=\left(h_{1}, \cdots, h_{n}\right)$ as $\mathcal{F}_{s, \boldsymbol{h}}=$ $\left[\boldsymbol{w}_{s}^{h_{1}}, \cdots, \boldsymbol{w}_{s}^{h_{n}}\right]$, Eq. (7) becomes

$$
\frac{1}{N_{\alpha}}\left(\mathcal{F}_{\alpha, j_{\alpha}}^{*} \otimes \boldsymbol{B}_{\Gamma, \alpha}\right) \hat{\boldsymbol{u}}_{\boldsymbol{j}_{\alpha}}^{(\alpha)}-\frac{1}{N_{\beta}}\left(\mathcal{F}_{\beta, j_{\beta}}^{*} \otimes \boldsymbol{B}_{\Gamma, \beta}\right) \hat{\boldsymbol{u}}_{\boldsymbol{j}_{\beta}}^{(\beta)}=0
$$


in which vectors $\hat{\boldsymbol{u}}_{\boldsymbol{h}}^{(s)}$ refer to the concatenation of vectors $\hat{\boldsymbol{u}}_{h_{i}}^{(s)}$ for all $h_{i} \in \boldsymbol{h}$. Now, let vectors of degreesof-freedom $\hat{\boldsymbol{u}}_{\boldsymbol{j}_{s}}^{(s)}$ be partitioned into boundary ones ${ }^{\Gamma} \hat{\boldsymbol{u}}_{\boldsymbol{j}_{s}}^{(s)}$ and internal ones ${ }^{i} \hat{\boldsymbol{u}}_{\boldsymbol{j}_{s}}^{(s)}$. Eq. (7b) can then be re-written as

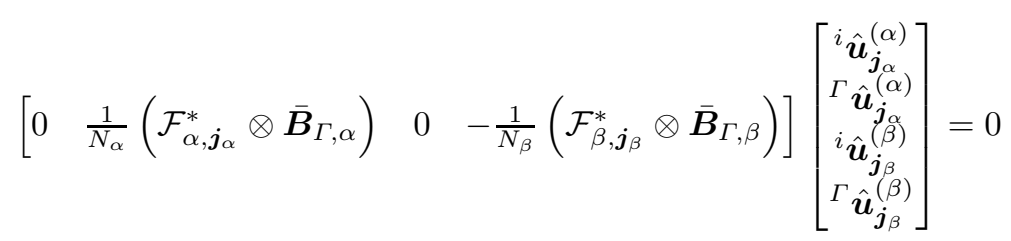

Eqs. (7) defines the coupling of cyclic components of adjacent stages $\alpha$ and $\beta$ associated with the fundamental cyclic harmonic $k$ of the multi-stage system. It should be noted that while the initial interface compatibility equation a priori involved coupling between all harmonics of the system (leading to no reduction), the final Eqs. (7) involves only a few coupled harmonics per stage (one or two in the example of Figs. 2) for each fundamental harmonic of the assembly.

\subsection{Multi-stage assembly}

Consider $\boldsymbol{k}=\left(k_{1}, \cdots, k_{n}\right)$ the set of cyclic harmonics to be retained in the analysis (usually defined by the analyst, depending on external forcing characteristics for example). For each retained cyclic harmonic, $k_{i}$ we define $\hat{\boldsymbol{q}}_{i}$, a unique set of degrees-of-freedom (in cyclic basis) in which redundant interfaces degrees-of-freedom have been eliminated.

$$
\left[\begin{array}{c}
\hat{\boldsymbol{u}}_{\boldsymbol{j}_{1}\left(k_{i}\right)}^{(1)} \\
\vdots \\
\hat{\boldsymbol{u}}_{\boldsymbol{j}_{S}\left(k_{i}\right)}^{(S)}
\end{array}\right]=\boldsymbol{L}_{k_{i}} \hat{\boldsymbol{q}}_{i}
$$

Matrix $\boldsymbol{L}_{k_{i}}$ can be derived from Eqs. (7) using a standard sub-structuring approach. Namely, based on Eq. (7c), the block portion of Eq. (8a) which concerns the coupling between sub-structures $\alpha$ and $\beta$ at interface $\Gamma$ is given by

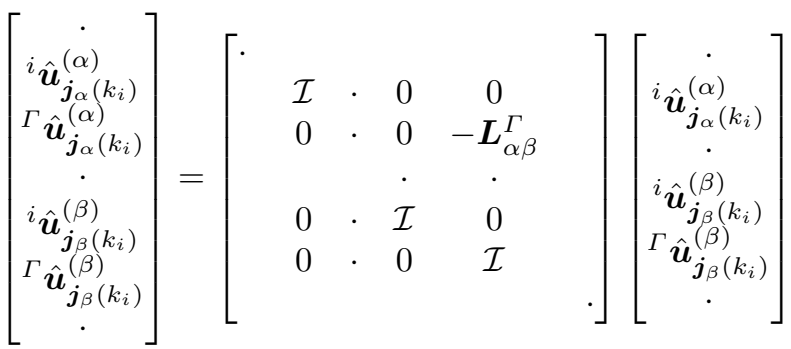

$$
\begin{aligned}
& \text { with } \quad \boldsymbol{L}_{\alpha \beta}^{\Gamma}=\frac{N_{\alpha}}{N_{\beta}}\left(\mathcal{F}_{\alpha, \boldsymbol{j}_{\alpha}\left(k_{i}\right)} \otimes \overline{\boldsymbol{B}}_{\Gamma, \alpha}^{+}\right)\left(\mathcal{F}_{\beta, j_{\beta}\left(k_{i}\right)}^{*} \otimes \overline{\boldsymbol{B}}_{\Gamma, \beta}\right)
\end{aligned}
$$

in which the superscript ${ }^{+}$refers to pseudo-inverse operation. The final equation of motion of the assembled system is then given by

$$
\tilde{\boldsymbol{M}}_{h_{i}} \ddot{\hat{\boldsymbol{q}}}_{i}+\tilde{\boldsymbol{C}}_{h_{i}} \dot{\hat{\boldsymbol{q}}}_{i}+\tilde{\boldsymbol{K}}_{h_{i}} \hat{\boldsymbol{q}}_{i}=\tilde{\boldsymbol{f}}_{i}
$$


in which

$$
\left\{\begin{aligned}
\tilde{\boldsymbol{K}}_{k_{i}} & =\boldsymbol{L}_{k_{i}}^{*} \operatorname{diag}\left(\operatorname{diag}\left(\boldsymbol{K}_{j}^{(s)}, j \in \boldsymbol{j}_{s}\left(k_{i}\right)\right), s \in \llbracket 1, S \rrbracket\right) \boldsymbol{L}_{k_{i}} \\
\tilde{\boldsymbol{C}}_{k_{i}} & =\boldsymbol{L}_{k_{i}}^{*} \operatorname{diag}\left(\operatorname{diag}\left(\boldsymbol{C}_{j}^{(s)}, j \in \boldsymbol{j}_{s}\left(k_{i}\right)\right), s \in \llbracket 1, S \rrbracket\right) \boldsymbol{L}_{k_{i}} \\
\tilde{\boldsymbol{M}}_{k_{i}} & =\boldsymbol{L}_{k_{i}}^{*} \operatorname{diag}\left(\operatorname{diag}\left(\boldsymbol{M}_{j}^{(s)}, j \in \boldsymbol{j}_{s}\left(k_{i}\right)\right), s \in \llbracket 1, S \rrbracket\right) \boldsymbol{L}_{k_{i}} \\
\tilde{\boldsymbol{f}}_{i} & =\boldsymbol{L}_{k_{i}}^{*}\left[\begin{array}{c}
\hat{\boldsymbol{f}}_{\boldsymbol{j}_{1}\left(k_{i}\right)}^{(1)} \\
\vdots \\
\hat{\boldsymbol{j}}_{\boldsymbol{j}_{S}\left(k_{i}\right)}^{(S)}
\end{array}\right]
\end{aligned}\right.
$$

Remark: when all fundamental harmonics are considered, higher harmonics are included as well due to the aliasing of Fourier series in accordance with the congruence rule of Eq. (6). In contrast with previous formulations of the multi-stage cyclic symmetry approach, the set of solutions obtained with the multi-stage cyclic symmetry method now remains admissible (i.e. no degenerated solutions appear and the number of eigensolutions of the multi-stage cyclic problem is strictly equal to the one of the reference problem).

\subsection{Multi-stage modal analysis and reduced-order model}

Based on the previous assembly procedure, the eigenproblem of the underlying autonomous and conservative multi-stage system can also be derived. It consists of sub-problems associated with each fundamental harmonic:

$$
\left(\tilde{\boldsymbol{K}}_{k}-\omega_{k}^{2} \tilde{\boldsymbol{M}}_{k}\right) \boldsymbol{X}_{k}=0 \quad \forall k \in \llbracket 0, N_{1}-1 \rrbracket
$$

in which matrices $\tilde{\boldsymbol{K}}_{k}$ and $\tilde{\boldsymbol{M}}_{k}$ are given according to Eqs. (10). Then introducing reduced matrices and vectors (identified by subscript $r$ )

$$
\left\{\begin{array}{l}
\boldsymbol{K}_{r}=\operatorname{diag}\left(\boldsymbol{X}_{k}^{*} \tilde{\boldsymbol{K}}_{k} \boldsymbol{X}_{k}, k \in \llbracket 0, N_{1}-1 \rrbracket\right) \\
\boldsymbol{C}_{r}=\operatorname{diag}\left(\boldsymbol{X}_{k}^{*} \tilde{\boldsymbol{C}}_{k} \boldsymbol{X}_{k}, k \in \llbracket 0, N_{1}-1 \rrbracket\right) \\
\boldsymbol{M}_{r}=\operatorname{diag}\left(\boldsymbol{X}_{k}^{*} \tilde{\boldsymbol{M}}_{k} \boldsymbol{X}_{k}, k \in \llbracket 0, N_{1}-1 \rrbracket\right) \\
\boldsymbol{f}_{r}=\boldsymbol{X}^{*}\left[\begin{array}{c}
\tilde{\boldsymbol{f}}_{0} \\
\vdots \\
\tilde{\boldsymbol{f}}_{N_{1}}
\end{array}\right] \quad \text { with } \quad \boldsymbol{X}=\operatorname{diag}\left(\boldsymbol{X}_{0}, \cdots, \boldsymbol{X}_{N_{1}}\right)
\end{array}\right.
$$

we can derive the reduced equation of motion of the multi-stage system (taking into account all harmonics):

$$
\boldsymbol{M}_{r} \ddot{\boldsymbol{\eta}}+\boldsymbol{C}_{r} \dot{\boldsymbol{\eta}}+\boldsymbol{K}_{r} \boldsymbol{\eta}=\boldsymbol{f}_{r}
$$

in which $\boldsymbol{\eta}$ is a vector of generalized (modal) coordinates.

\section{Stochastic reduced-order model}

As mentioned in the introduction, it is now common to model mistuned systems using reduced-order models based on modal synthesis. In this investigation, a technique called Component Mode Mistuning method originally proposed by Lim et al. [13] is used. A global system (the rotor) with local components (blades) is considered in a substructuring-like framework. The response of the uncertain system is obtained from a perturbation of the underlying tuned system's model. Uncertainty is assumed to be concentrated in components and is quantified in terms of their modal properties. Uncertainty quantification in the modal space offers several advantages [8] among which a reduction of the number of uncertain parameters and 
the ability to account for all sources of uncertainty (parametric and non-parametric). Accordingly, the key point of this approach lies in its ability to propagate uncertainty from the components' modal space to the global modal perturbational reduced-order model using a projection technique $[3,13]$ and without actually requiring any physical substructuring.

\subsection{Perturbational reduced-order model formulation}

Consider the previously defined reduced modal basis $\boldsymbol{X}$, obtained by solving each sub-problems (11) and reduced matrices defined in Eqs. (12). Then, consider components (i.e. parts) of the system with uncertain characteristics and which structural matrices (i.e. restrictions of global matrices associated with respective components degrees-of-freedom) are random. For the multi-stage system of bladed disks, such components refer to each blade of stage $s$, physical displacement vector of which can be given as:

$$
\boldsymbol{u}^{(s, c)}=\left[\begin{array}{c}
\boldsymbol{u}^{\left(s, c_{0}\right)} \\
\vdots \\
\boldsymbol{u}^{\left(s, c_{N_{s}-1}\right)}
\end{array}\right]=\mathcal{F}_{s}^{*} \otimes \boldsymbol{X}^{(s, c)} \boldsymbol{\eta} \quad \text { with } \quad \mathcal{F}_{s}=\left[\mathcal{F}_{s, \boldsymbol{j}(0)}, \ldots, \mathcal{F}_{s, \boldsymbol{j}\left(N_{1}-1\right)}\right]
$$

in which $\boldsymbol{u}^{\left(s, c_{n}\right)}$ represents the displacement vector of blade $n$ of stage $s$ and $\boldsymbol{X}^{(s, c)}$ is the restriction of cyclic eigenvectors matrix to blade's degrees-of-freedom of stage $s$. Fourier matrices $\mathcal{F}_{s, \boldsymbol{j}(k)}$ consist of the concatenation of Fourier vectors of stage $s$ cyclic basis associated the definition of fundamental harmonic $k$ (note that matrix $\mathcal{F}_{s}$ is actually the full Fourier matrix but with columns ordered according to the fundamental harmonics definition).

The perturbational reduced-order model is defined by generalized matrices with these random matrices projected in the base-line model's basis $(\boldsymbol{A}$ can refer to either $\boldsymbol{K}, \boldsymbol{C}$ or $\boldsymbol{M})$ :

$$
\boldsymbol{A}_{r}^{\prime}=\boldsymbol{A}_{r}+\sum_{s=1}^{S}\left(\mathcal{F}_{s}^{*} \otimes \boldsymbol{X}^{(s, c)}\right)^{*} \operatorname{diag}\left(\delta \boldsymbol{A}^{\left(s, c_{n}\right)}, n \in \llbracket 0, N_{s}-1 \rrbracket\right)\left(\mathcal{F}_{s}^{*} \otimes \boldsymbol{X}^{(s, c)}\right)
$$

in which $\delta \boldsymbol{A}^{\left(s, c_{n}\right)}$ is a random deviation matrix from the mean matrix $\left\langle\boldsymbol{A}^{\left(s, c_{n}\right)}\right\rangle$ of each blade. This projection is valid as long as $\left\|\delta \boldsymbol{A}^{\left(s, c_{n}\right)}\right\| \ll\left\|\left\langle\boldsymbol{A}^{\left(s, c_{n}\right)}\right\rangle\right\|$.

In the following, quantification of uncertainty will be assessed in the components modal space (distinct from the system modal space).

\subsection{Introduction of uncertainty and projection}

As we aim at introducing and quantifying uncertainties of components in their modal properties, a CraigBampton reduction is introduced. Namely, let us consider a component $c$ and denote $\boldsymbol{u}_{b}^{(c)}$ and $\boldsymbol{u}_{i}^{(c)}$ vectors corresponding boundary and internal degrees-of-freedom respectively. The latter can be expressed as

$$
\boldsymbol{u}_{i}^{(c)}=\boldsymbol{\Psi} \boldsymbol{\eta}_{b}^{(c)}+\boldsymbol{\Phi} \boldsymbol{\eta}_{i}^{(c)}
$$

where $\boldsymbol{\eta}_{b}^{(c)}=\boldsymbol{u}_{b}^{(c)}$ are generalized coordinates vectors which correspond to static modes:

$$
\boldsymbol{\Psi}=-\boldsymbol{K}_{i i}^{(c)}{ }^{-1} \boldsymbol{K}_{i b}^{(c)}
$$

and $\boldsymbol{\eta}_{i}^{(c)}$ are generalized coordinates associated with fixed interface normal modes such as:

$$
\boldsymbol{\Phi}^{T} \boldsymbol{M}_{i i}^{(c)} \boldsymbol{\Phi}=\mathcal{I} \quad \text { and } \quad \boldsymbol{\Phi}^{T} \boldsymbol{K}_{i i}^{(c)} \boldsymbol{\Phi}=\boldsymbol{\Lambda}^{(c)}
$$

Now uncertainty is quantified as the perturbation random matrix $\delta \boldsymbol{\Lambda}^{(c)}$. The next step is to propagate uncertainty in the global modal matrices defined in Eqs. (15). Using the orthogonality relations (18) along with Eq. (17), Eq. (16) leads to:

$$
\boldsymbol{\eta}_{i}^{(c)}=\boldsymbol{\Lambda}^{(c)^{-1}} \boldsymbol{\Phi}^{T} \boldsymbol{K}_{i b}^{(c)} \boldsymbol{u}_{b}^{(c)}+\boldsymbol{\Phi}^{T} \boldsymbol{M}_{i i}^{(c)} \boldsymbol{u}_{i}^{(c)}=\boldsymbol{P} \boldsymbol{u}^{(c)}
$$


Using projection matrix $\boldsymbol{P}$ defined in Eq. (19), one can now express perturbation stiffness matrices $\delta \boldsymbol{K}^{\left(s, c_{n}\right)}$ in Eqs. (15) as a function of $\delta \boldsymbol{\Lambda}^{(c)}$ :

$$
\boldsymbol{K}_{r}^{\prime}=\boldsymbol{K}_{r}+\sum_{s=1}^{S}\left(\mathcal{F}_{s}^{*} \otimes \boldsymbol{X}^{(s, c)}\right)^{*} \operatorname{diag}\left(\boldsymbol{P}^{T} \delta \boldsymbol{\Lambda}^{\left(s, c_{n}\right)} \boldsymbol{P}, n \in \llbracket 0, N_{s}-1 \rrbracket\right)\left(\mathcal{F}_{s}^{*} \otimes \boldsymbol{X}^{(s, c)}\right)
$$

As mentioned earlier, this representation of uncertainties as perturbations of components' eigenvalues results in either a perturbation of the stiffness or mass matrix of the global system (here, the stiffness matrix is chosen). Also, if a modal damping definition is considered the perturbation damping matrix can be treated as well by introducing a random modal damping matrix.

The resulting stochastic reduced-order model contains only the modes of the base-line tuned system. The number of component modes is the main parameter that controls the convergence and accuracy of the reduced-order model. The key point is that the size of the model is not affected by this parameter. Also note that the projection matrix $\boldsymbol{P}$ in Eq. (19) needs to be calculated only once so that a relatively small number of operations are necessary in iterative stochastic solvers.

\subsection{Modelling of uncertainties}

Introducing uncertainties directly in the modal space significantly reduces the number of parameters to be identified but does not really solve the problem of their modelling. As the previously defined stochastic reduced-order model is quite generic, it can be used in conjunction with various kinds of probabilistic models of uncertainties, provided that resulting perturbations remain sufficiently small. A straightforward and popular approach (in mistuning applications at least) is to consider only one scalar uncertain parameter per blade, namely the Young modulus. Resulting perturbation matrices $\delta \Lambda^{(c)}$ are then diagonal and proportional to the latter. Clearly, generic perturbations (expressed as random structural matrices) will lead to full random modal matrices $\delta \Lambda^{(c)}$. However, when perturbations are sufficiently small (i.e. when perturbations of components eigenvectors are of second-order), it is very likely that they will feature dominant diagonal terms. As a consequence, it seems legitimate to directly consider random diagonal matrices in which eigenvalues are random parameters. Also note that this is consistent with the assumption that system and component modes of the stochastic model are equal to their deterministic counterpart. Then, probabilistic specifications of these random variables can be assessed based on available experimental data (mistuning identification techniques have been developed for this purpose [7, 9, 12]). In [14, 16], the issue of identifying a probability model for these random variables has been discussed and a technique based on the maximum likelihood principle has been proposed in particular. In the absence of experimental information, statistical characteristics of random eigenvalues would have to be assessed a priori. In this case, considering eigenvalues as independent parameters characterized by a Gaussian distribution is a natural choice (which will be made in the applications that follow). Note however that, depending of the type of perturbation involved, eigenvalue parameters may actually feature some correlation which could for example be assessed by means of sensitivity analyses. Finally, the most general (yet not trivial) approach currently available seems to be the non-parametric modelling technique $[4,5]$ which makes use of the information theory and maximum entropy principle in the framework of random matrices theory.

\section{Applications to a two-stages bladed rotor}

\subsection{Model description}

We consider a bladed rotor consisting of two HP-compressor integrally (i.e. single-piece made) bladed disks composed of 36 and 60 sectors respectively. Finite-element meshes of elementary sectors are shown in Fig. 3a along with their respective blade component in Fig. 3b. Fig. 4 displays the modal situation of this assembly as calculated by multi-stage cyclic symmetry (Fig. 4a) compared with a fixed inter-stage interface situation (Fig. 4b) in the form of a cyclic harmonics / (normalized) frequency diagram. Grey levels of dots refer to the 


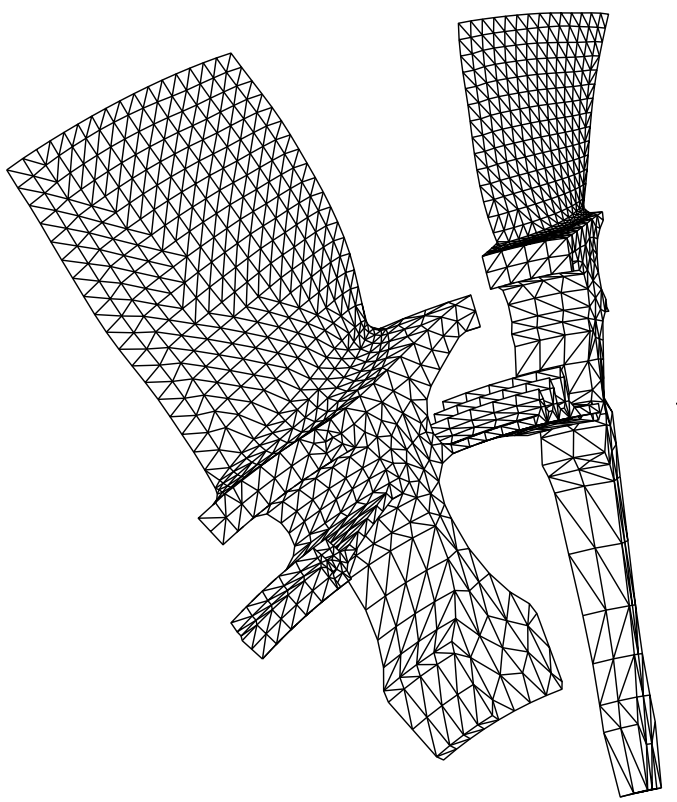

(a)

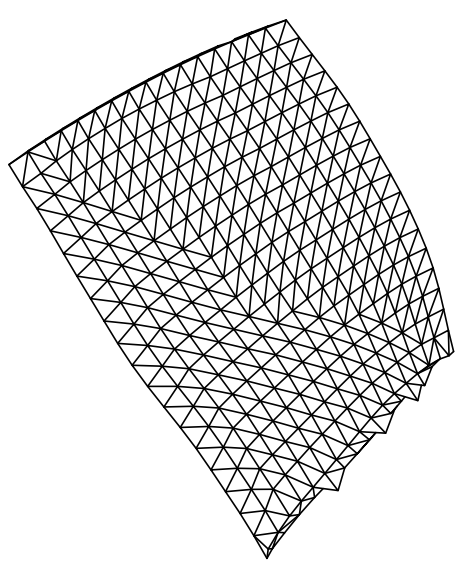

(b)

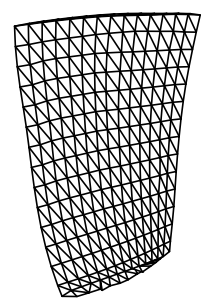

Figure 3: Finite element mesh of the multi-stage assembly (a) and blade components of each stage (b)

level of participation of each stage in the global motion. This modal participation is obtained by calculating modal masses for each stage respectively. White dots refer to a 1-st stage dominated motion and black dots to a 2-nd stage dominated motion, intermediate greys refer to coupled multi-stage modes. Comparing the two plots, strong couplings between stages appear in particular in lower harmonics of in regions of frequency veering.

\subsection{Modal analysis}

In this section, the validity and accuracy of the proposed reduced-order modelling approach are investigated. Comparisons and correlation results from modal analyses of reduced-order models and of a reference $360^{\circ}$ model are considered for this purpose. A proportional mistuning model is here considered. A set of normally distributed random parameters corresponding to the Young modulus of each blade is generated. Samples have zero mean and their standard deviation corresponds to the so-called mistuning amplitude.

The following examples focus on the frequency region from $168 \mathrm{UF}$ (unit of frequency) to 186 UF (with reference to Fig. 4). There are 39 modes in this frequency range, which defines the size of the reducedorder model. Comparisons of frequencies and mode shapes are proposed for two random mistuning patterns with amplitude of $3 \%$ and $8 \%$ respectively in Figs. 5 and 6 . Relative frequency errors remain below $1 \%$ for both cases and modal correlation is also very satisfying. While the $8 \%$ case shows small discrepancies in modes pairing, correlation remains acceptable. The convergence limit of the reduced-order model seems to be around $10 \%$ of mistuning. Note that typical amplitudes of random mistuning are generally less than $5 \%$.

Finally, we consider in Figs. 7 three mode shapes in the frequency range 355 - 386 UF (see Fig. 4) without and with mistuning and investigate the combined effects of mistuning and multi-stage coupling on localization. The first one, Figs. 7 a, corresponds to a 4 nodal diameter mode of the tuned system and features significant coupling between stages. Blades of the first and second stages experience complex torsion and second bending motions respectively. Figs. $7 \mathrm{~b}$ and $7 \mathrm{c}$ are mode shapes of the system with $5 \%$ random mistuning pattern on both stages. Due to mistuning, these mode shapes feature localization of the vibratory energy either on a few blades of one stage (Fig. 7b) or on a few blades of both stages (Fig. 7c). Hence, while 


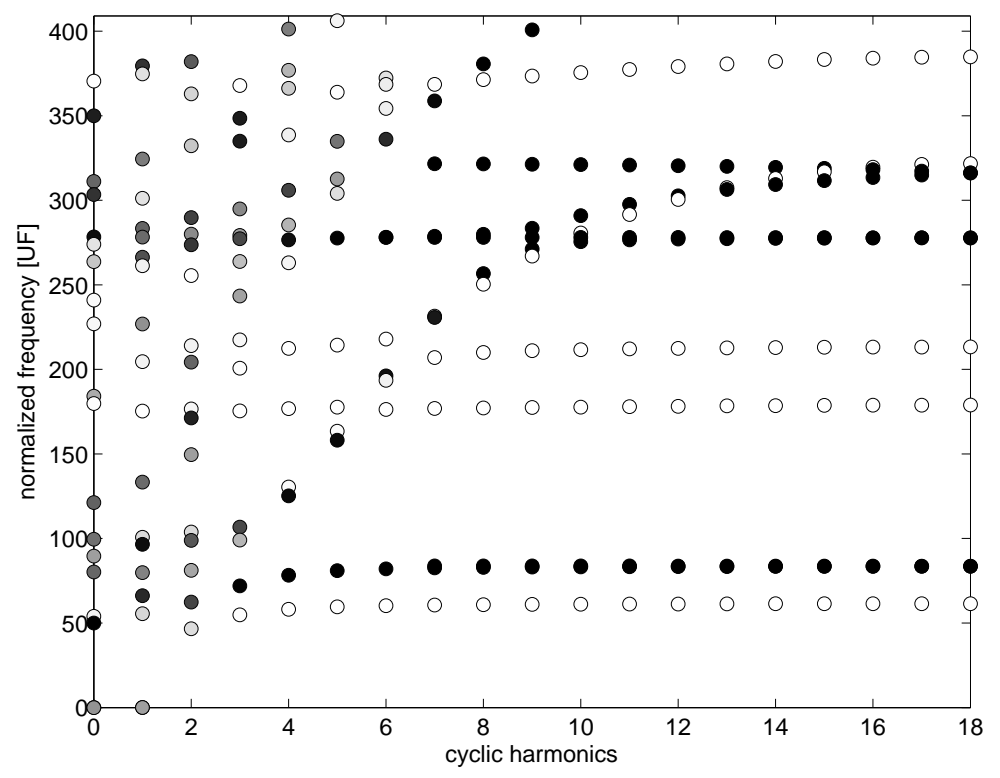

(a)

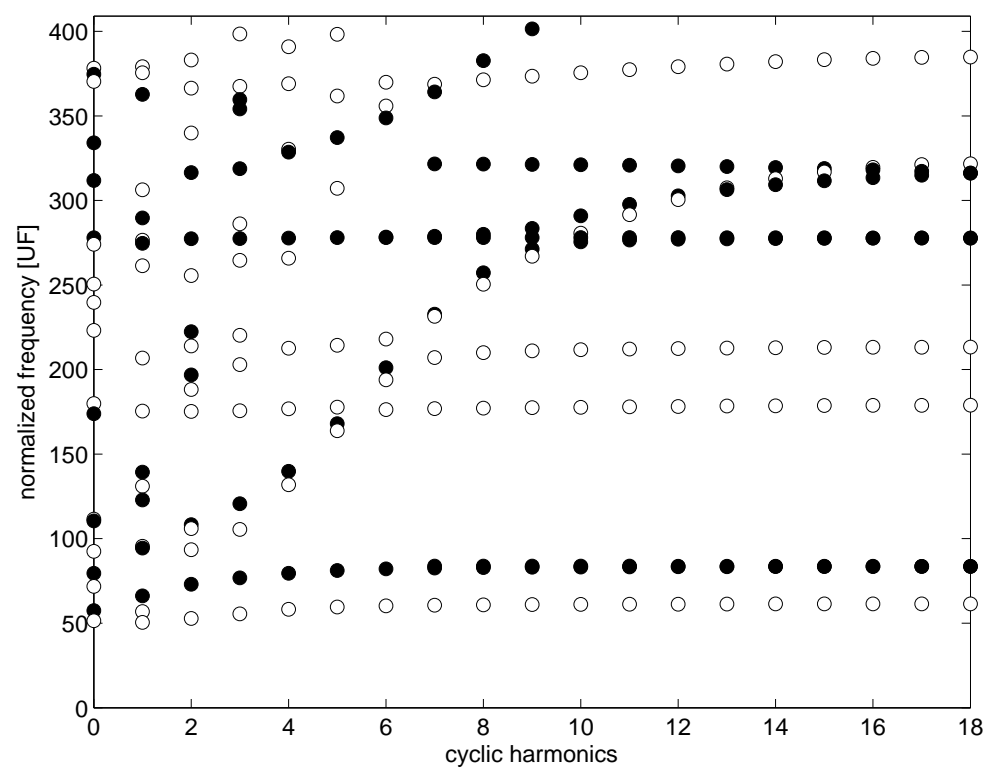

(b)

Figure 4: Cyclic harmonics / (normalized) eigenfrequency plots with fixed interstage interface (b) and multistage cyclic symmetry (a); grey levels account for modal participations of each stage (white: stage 1, black: stage 2) 


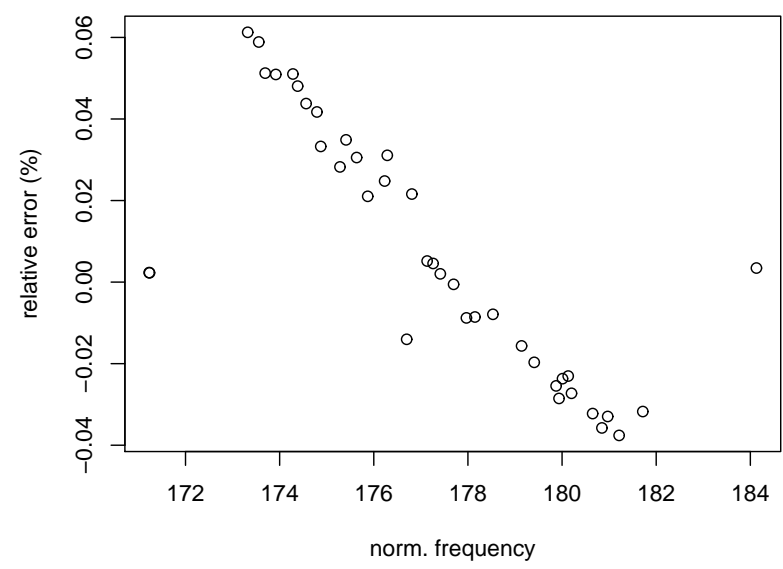

(a)

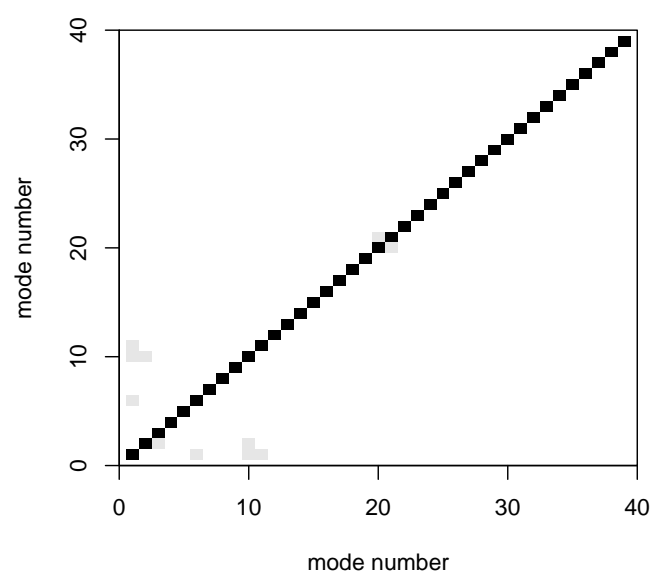

(b)

Figure 5: Modal correlation in presence of a $3 \%$ random mistuning pattern on each stage; (a) frequency error and (b) Modal Assurance Criterion

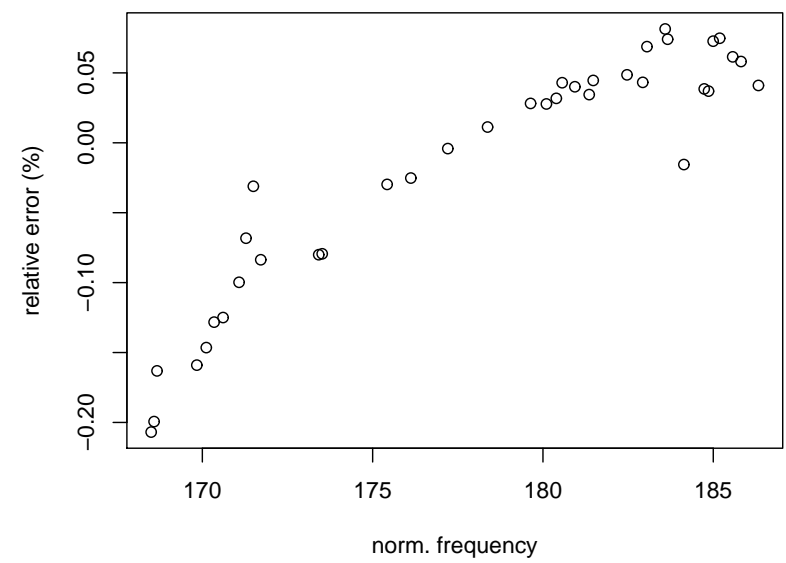

(a)

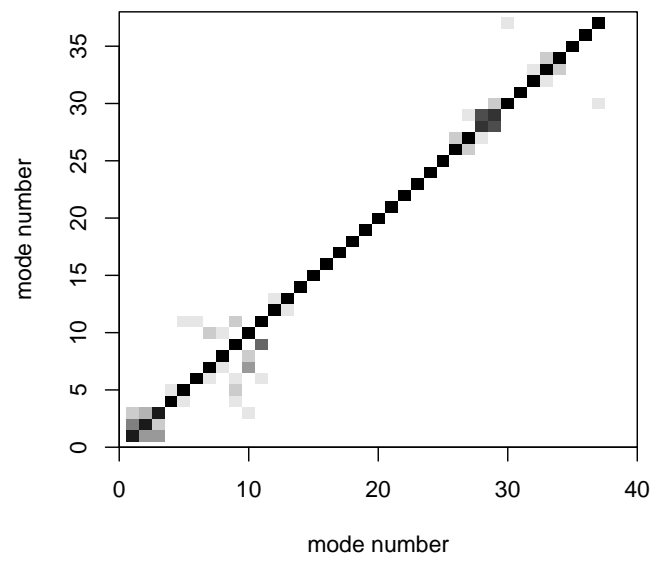

(b)

Figure 6: Modal correlation in presence of a $8 \%$ random mistuning pattern on each stage; (a) frequency error and (b) Modal Assurance Criterion 


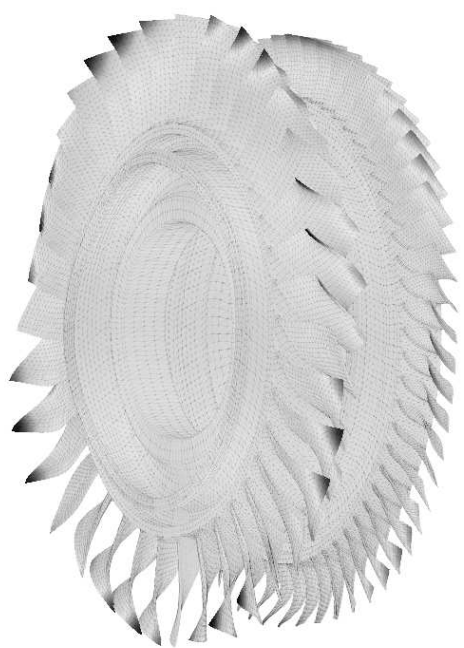

(a)

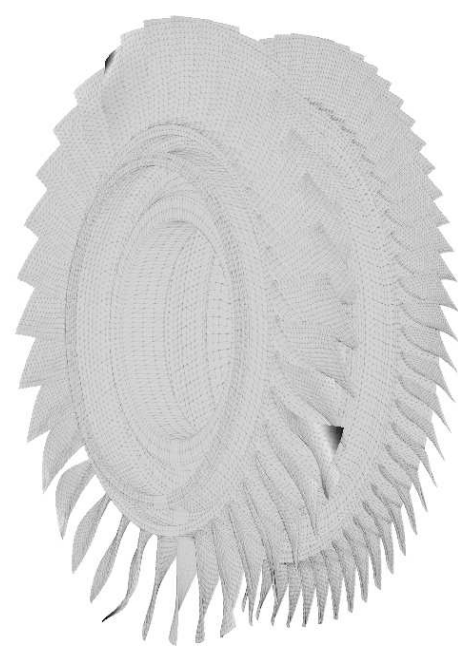

(b)

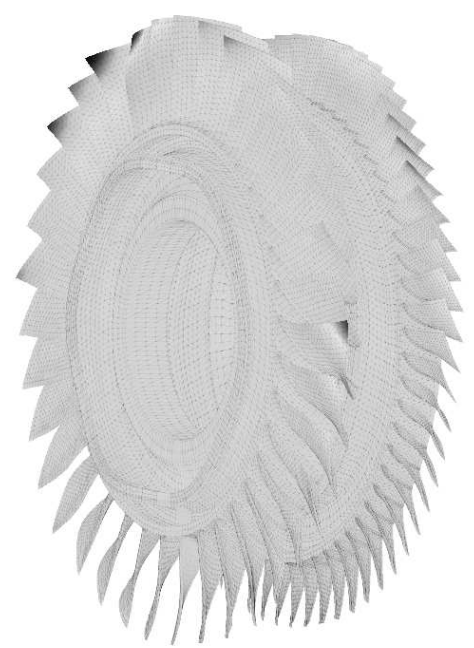

(c)

Figure 7: Examples of mode shapes of the multi-stage system; (a) tuned mode at 366 UF (4-ND), (b) and (b) mistuned modes at $356 \mathrm{UF}$ and $369 \mathrm{UF}$.

it is obvious from Fig. 7a that accounting for the multi-stage coupling in the tuned case is important, the two last examples demonstrate that it is still important for mistuned structures since multi-stage motions are detected despite mistuning.

\subsection{Convergence analyses}

In this section, we investigate the convergence of the stochastic reduced-order model with respect to various analyses and modelling parameters. The statistics of interest are the quadratic mean and various percentile values of the magnification of forced responses due to mistuning. Magnification is defined as the ratio which compares forced responses maxima with respect to all degrees-of-freedom and frequencies of mistuned systems and with those of the underlying tuned system.

In this section and of the following one, the system is forced by two single-point (top leading-edge blade's node) harmonic excitations of harmonics 4 an 3 for stage 1 and 2 respectively. Excitation amplitudes are 1 and 0.6 in stage 1 and 2 respectively (roughly proportional to blades area). Uncertainty is introduced in the form of random diagonal eigenvalue matrices $\delta \boldsymbol{\Lambda}^{(c)}$ for each uncertain component $c$. We then have to quantify $n_{c}$ random parameters (component mode eigenvalues) per uncertain component (blade). Without any a priori information, these random parameters can be assumed to follow a normal distribution, which standard deviation defines the mistuning amplitude (per component mode). Correlation between eigenvalues is probably more difficult to assess and without any available information, it will be assumed that these random variables are uncorrelated.

First, we consider convergence with respect to model reduction parameters. The stochastic reduced-order model is defined by two main reduction parameters: (1) the number of system modes and (2) the number of component modes. The first parameter mainly depends on the frequency band of interest, which is usually quite narrow (resonances close to engine order crossings in a Campbell diagram). However, as mistuning may trigger resonances from modes outside of the target region the number of system modes usually has to be increased to include neighbouring modes. Based on this consideration, the required number of system modes can be assessed empirically. As discussed earlier, the number of component modes - which are the inputs for uncertainty quantification - is the main parameter which determines the accuracy and convergence of 
the reduced-order model. In order to investigate the effects of truncation or partial selection of component modes, let us compare the statistics (quadratic mean here) of forced response amplification of both stages obtained by either (a) selecting only one component mode per stage and (b) including ten component modes per stage. The latter (reference solution) is obtained by considering all component modes eigenvalues as independent random variables (leading to a diagonal, but non-proportional, matrix $\delta \boldsymbol{\Lambda}^{(c)}$ ) whereas, in the former, there is only one uncertain parameter per blade.

Figs. 8 illustrate the comparison in the frequency range 350-400 UF (see Figs. 4). It is clear that in

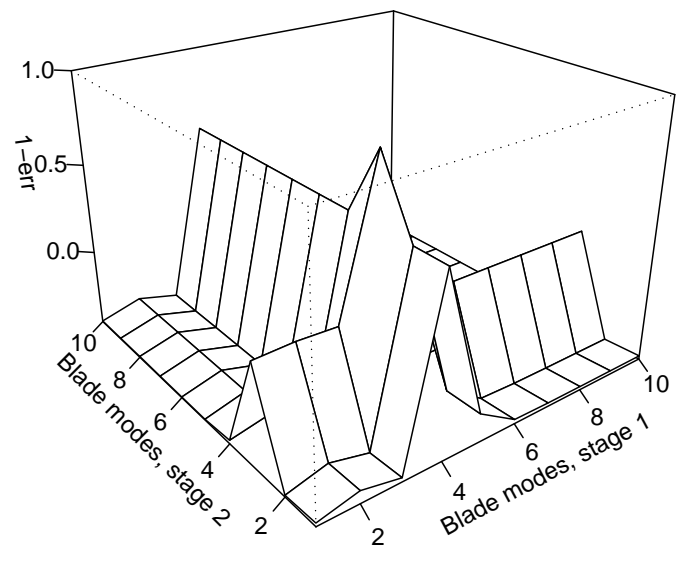

(a)

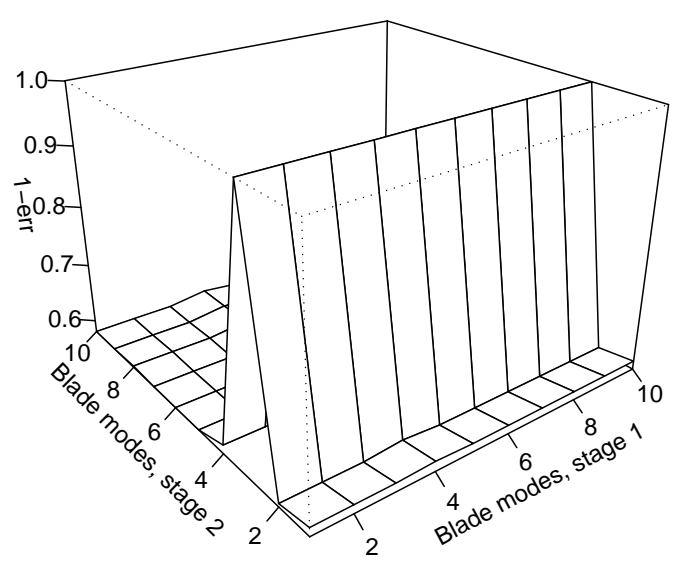

(b)

Figure 8: Effects of individual component modes participations on forced response amplification. Error in quadratic mean of forced response amplification of stage 1 (a) and 2 (b) with each component (blade) modes separately as compared with all modes.

this frequency range, dominant component modes are mode 4 (and slightly mode 2 ) for stage 1 and mode 3 for stage 2 . In other words, perturbations of at most one component mode per stage affect the forced response amplification statistics so that considering mode 4 and 3 for stage 1 and 2 respectively as uncertain parameters is sufficient. This greatly simplifies the uncertainty quantification step of the analysis since it now reduces to defining $N_{s}$ independent parameters per stage. Similar conclusions may be found for other (narrow) frequency ranges.

Second, we consider the convergence with respect with the number of samples in Monte Carlo simulations. As an example, Figs. 9 show the evolution of the aforementioned criteria (mean and percentiles) as a function of the number of samples in Monte Carlo simulations. Above about 500 samples, all convergence criteria appear to be stabilized. Similar average numbers of samples were found for other frequency ranges of interest.

\subsection{Effects of multi-stage coupling on mistuning sensitivity}

This section is devoted to mistuning sensitivity analyses based on the representative two-stages rotor model presented in Section 5.1. In particular, we investigate the effects of multi-stage coupling on the forced response amplification due to mistuning introduced in each stage separately. Statistics of amplification in case of multi-stage coupling are compared with those of isolated stages situations (i.e. with the interstage boundary clamped). With reference to Fig. 4, we consider two frequency ranges, namely 250-290 UF and 340-395 UF, which feature significant influence of the multi-stage coupling and concern one family of modes for stage 2 and 1, respectively. Monte-Carlo simulations are used, with 500 mistuning samples with $3 \%$ of amplitude for each configurations. As discussed in the previous section, since narrow frequency ranges are 


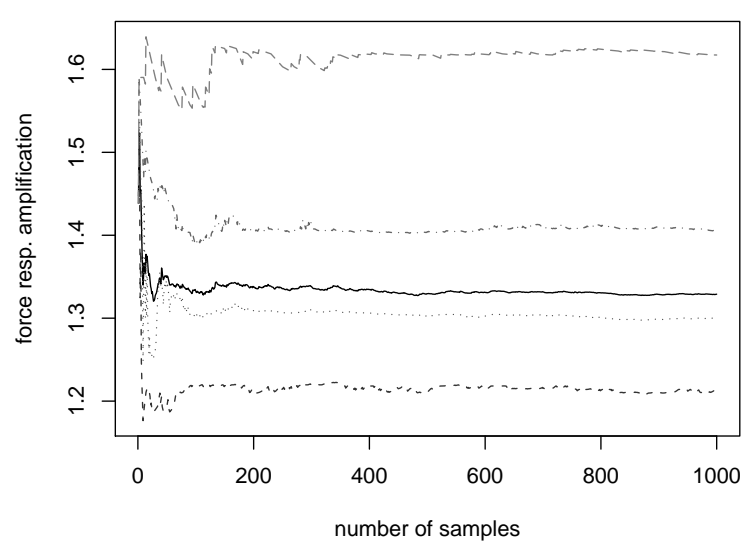

(a)

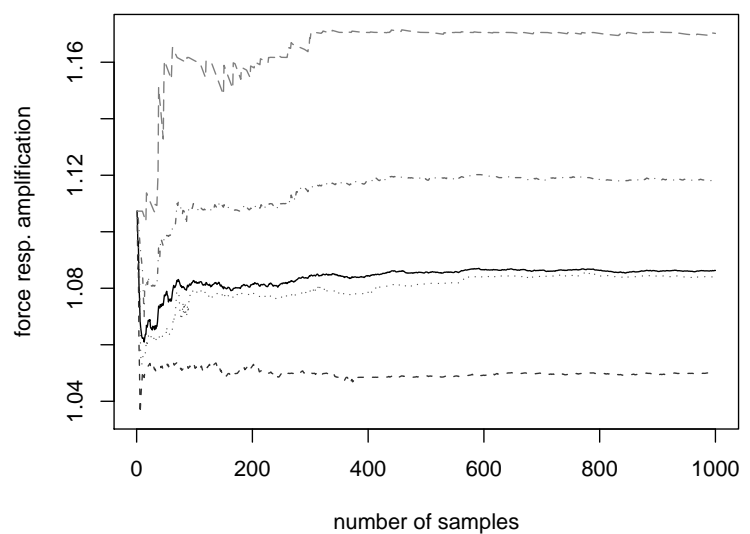

(b)

Figure 9: Convergence for forced response statistics with respect to the number of samples in Monte Carlo simulations; (a) stage 1 and (b) stage 2; solid line: mean; dashed lines: 0.25, 0.5, 0.75, 0.95 percentiles.

considered, it is sufficient to consider only one random component eigenvalue per blade (mode 2 in stage 2 for frequency range 1 and mode 4 in stage 1 for frequency range 2).

Histograms and empirical cumulative distribution functions of forced response amplification are presented in Figs. 10 for the two frequency ranges respectively. Effects of multi-stage coupling are clearly different depending on the modal family of interest:

- For the first family, in Fig. 10a, the multi-stage coupling tends to increase the amplification in stage 2 (mean and maximum amplifications increase from 1.37 and 1.70 to 1.61 and 2.2 respectively) but leads to a diminution of forced response in stage 1 (where the mean and maximum amplifications reach 0.83 and 1.25 respectively).

- Conversely, in the second family (Fig. 10b), the interstage coupling has almost no effects on amplification statistics in the first stage. Propagation of disorder from the first to the second stage has very little effects on the dynamic response of the latter (mean of amplification is 0.99 and standard deviation is $0.017)$.

In the first case, multi-stage coupling mostly results in increasing disk flexibility, which in turns, induces veering phenomena due to a higher modal density in the considered excitation configuration. The latter are of primary importance regarding mistuning sensitivity [2] and are often responsible for such an increase of amplification. On the other hand, the second case illustrates multi-stage coupling can also have very little effects on forced response amplification in some cases. While the above results clearly depend on the considered case-study, they however show that the effects of inter-stage coupling on mistuning sensitivity cannot be assessed a priori and thus demonstrate the importance of a proper assessment of multi-stage coupling in mistuning analyses.

\section{Conclusions}

A methodology for modelling and analysis of multi-stage systems of mistuned bladed disks is proposed. It is based on the perturbation of a reduced order model built using modal synthesis. The underlying modal basis 


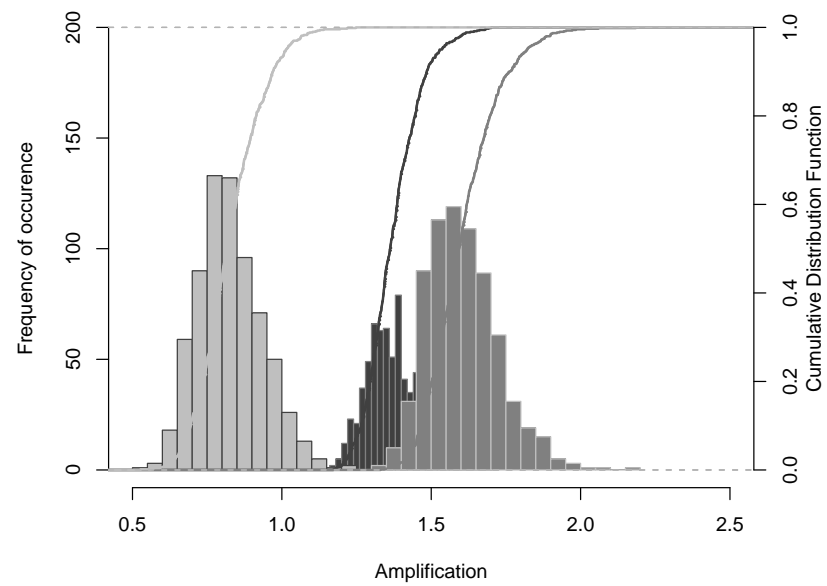

(a)

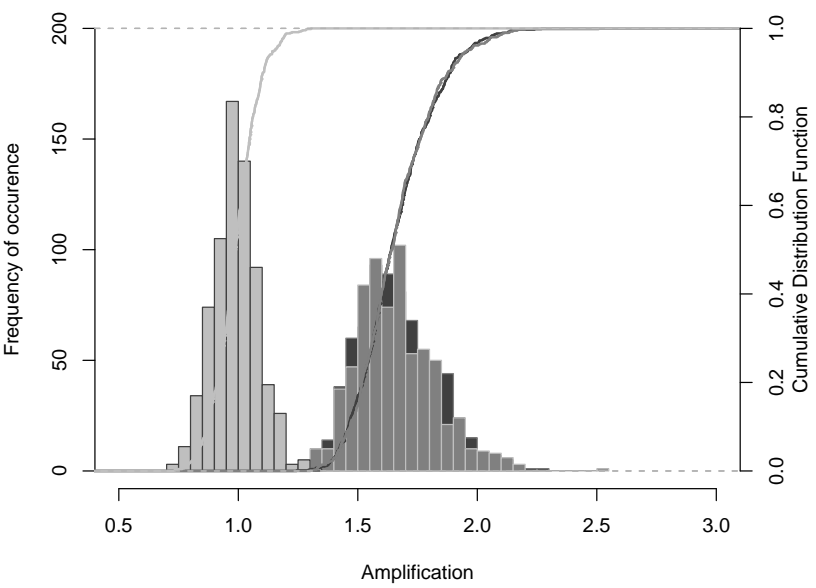

(b)

Figure 10: Influence of multi-stage coupling on mistuning sensitivity. (a), forced response amplification in frequency range 250-290 UF with mistuning on the second stage only: ( $\left({ }^{2}\right)$ stage 2 isolated and ( $\square$ ) stage 2 and ( $(-)$ stage 1 with inter-stage coupling. (b), forced response amplification in frequency range 340-386 UF with mistuning on the first stage only; ( $\square$ ) stage 1 isolated and $(\square)$ stage 1 and $(\square)$ stage 2 with inter-stage. 
is derived using the multi-stage cyclic symmetry approach which has been reviewed and extended in this paper. It makes it possible to represent a multi-stage system of cyclic structures based on their respective fundamental sector as in traditional cyclic symmetry. The resulting reduced order model is very compact, hence suitable for statistical analyses, and features satisfying accuracy for small to moderate perturbation amplitudes. Also, uncertainty can be introduced in the modal space at the component level which makes their quantification easier.

Applications and uncertainty analyses based on a representative two-stages rotor are also proposed and demonstrate (1) the efficiency of the approach and (2) the importance of considering inter-stage coupling in mistuning analysis.

\section{Acknowledgement}

Thanks go to Snecma for its technical and financial support. This work takes place in the framework of the MAIA mechanical research and technology program sponsored by CNRS, ONERA and SAFRAN Group.

\section{References}

[1] R. Bladh, M. P. Castanier, and C. Pierre. "Component-Mode based reduced order modelling techniques for mistuned bladed disks - Part 1: Theorical models". In: Journal of Engineering for Gas Turbines and Power 123 (2001), pp. 89-108. DOI: 10.1115/1.1338947.

[2] R. Bladh, M. P. Castanier, and C. Pierre. "Effects of Multistage Coupling and Disk Flexibility on Mistuned Bladed Disk Dynamics". In: Journal of Engineering for Gas Turbines and Power 125 (2003), pp. 121-130. DOI: 10.1115/1.1498267.

[3] R. Bladh, M. P. Castanier, and C. Pierre. "Reduced Order Modeling and Vibration Analysis of Mistuned Bladed Disk Assemblies with Shrouds". In: Journal of Engineering for Gas Turbines and Power 121 (1999), pp. 515-522. DOI: 10.1115/1.2818503.

[4] E. Capiez-Lernout and C. Soize. "Nonparametric Modeling of Random Uncertainties for Dynamic Response of Mistuned Bladed Disks". In: Journal of Engineering for Gas Turbines and Power 126.3 (2004), p. 610. DOI: 10.1115/1.1760527.

[5] E. Capiez-Lernout, C. Soize, J. P. Lombard, C. Dupont, and E. Seinturier. "Blade Manufacturing Tolerances Definition for a Mistuned Industrial Bladed Disk". In: Journal of Engineering for Gas Turbines and Power 127.3 (2005), pp. 621-628. DOI: 10.1115/1.1850497.

[6] M. P. Castanier and C. Pierre. "Modeling and Analysis of Mistuned Bladed Disk Vibration: Status and Emerging Directions". In: Journal of propulsion and power 22.2 (2006), pp. 384-396.

[7] D. M. Feiner and J. H. Griffin. "Mistuning Identification of Bladed Disks Using a Fundamental Mistuning Model-Part I: Theory". In: Journal of Turbomachinery 126.1 (2004), pp. 150-158. DOI: 10.1115/1.1643913.

[8] L. Hinke, F. Dohnal, B. R. Mace, T. P. Waters, and N. S. Ferguson. "Component mode synthesis as a framework for uncertainty analysis". In: Journal of Sound and Vibration 324.1-2 (2009), pp. 161-178. DOI: $10.1016 / j \cdot j s v \cdot 2009.01 .056$.

[9] J. Judge, C. Pierre, and S. L. Ceccio. "Experimental Mistuning Identification in Bladed Disks Using a Component-Mode-Based Reduced-Order Model". In: AIAA Journal 47.5 (2009), pp. 1277-1287. DoI: $10.2514 / 1.41214$.

[10] D. Laxalde, J.-P. Lombard, and F. Thouverez. "Dynamics of multi-stage bladed disks systems". In: Journal of Engineering for Gas Turbines and Power 129.4 (2007), pp. 1058-1064. DOI: 10.1115/1.2747641. OAI: hal.archives-ouvertes.fr:hal-00243153. 
[11] D. Laxalde, F. Thouverez, and J.-P. Lombard. "Dynamical analysis of multi-Stage cyclic structures". In: Mechanics Research Communications 34.4 (2007), pp. 379-384. DOI: 10.1016/j.mechrescom.2007.02.004. OAI: hal.archives-ouvertes.fr:hal-00227538.

[12] D. Laxalde, F. Thouverez, J.-J. Sinou, J.-P. Lombard, and S. Baumhauer. "Mistuning identification and model updating of an industrial blisk". In: International Journal of Rotating Machinery vol. 2007 (2007), Article ID 17289, 10 pages. DOI: 10.1155/2007/17289. OAI: hal.archives-ouvertes.fr:hal-00214219.

[13] Sang-Ho Lim, Ronnie Bladh, Matthew P. Castanier, and Christophe Pierre. "Compact, Generalized Component Mode Mistuning Representation for Modeling Bladed Disk Vibration". In: AIAA Journal 45.9 (2007), p. 2285. DOI: $10.2514 / 1.13172$.

[14] M. P. Mignolet, A. J. Rivas-Guerra, and J. P. Delor. "Identification of Mistuning Characteristics of Bladed Disks From Free Response Data-Part I". In: Journal of Engineering for Gas Turbines and Power 123.2 (2001), pp. 395-404. DOI: 10.1115/1.1338949.

[15] M. Nikolic. "New Insights into the Blade Mistuning Problem". PhD thesis. Imperial College London, University of London, 2006.

[16] A. J. Rivas-Guerra, M. P. Mignolet, and J. P. Delor. "Identification of Mistuning Characteristics of Bladed Disks From Free Response Data - Part II". In: Journal of Engineering for Gas Turbines and Power 123.2 (2001), pp. 404-411. DOI: 10.1115/1.1338950.

[17] D. L. Thomas. "Dynamics of rotationally periodic structures". In: International Journal for Numerical Methods in Engineering 14.1 (1979), pp. 81-102. DOI: 10.1002/nme.1620140107.

[18] S. T. Wei and C. Pierre. "Localization phenomena in mistuned assemblies with cyclic symmetry Part 1: Free Vibrations". In: Journal of Vibration, Acoustics, Stress, and Reliability in Design 110 (1988), pp. 429-437.

[19] S. T. Wei and C. Pierre. "Localization phenomena in mistuned assemblies with cyclic symmetry Part 2: Forced Vibrations". In: Journal of Vibration, Acoustics, Stress, and Reliability in Design 110 (1988), pp. 439-449.

[20] M. T. Yang and J. H. Griffin. "A reduced-Order model of mistuning using a subset of nominal system modes". In: Journal of Engineering for Gas Turbines and Power 123 (2001), pp. 893-900. DoI: 10.1115/1.1385197. 